ISSN 1112-9867

Available online at

http://www.jfas.info

\title{
THE EVOLUTION OF SHRINKAGE STRAIN OF PET-MORTAR COMPOSITE
} ECO-MATERIALS

O. Chaib ${ }^{1}$, A.S. Benosman ${ }^{1,2,3^{*}}$, N. Kazi Tani ${ }^{2,4}$, Y. Senhadji ${ }^{1}$, M. Mouli ${ }^{1}$, H. Taïbi ${ }^{3}$ and M. Hamadache $^{1}$

${ }^{1}$ Département de Génie Civil, Laboratoire des Matériaux, ENPO d'Oran, Algérie. ${ }^{2}$ École Supérieure des Sciences Appliquées (ESSAT), Bel Horizon, Tlemcen 13000, Algérie. ${ }^{3}$ Faculté des Sciences Exactes et Appliquées, Lab. LCP, Univ.d'Oran 1, A. Benbella, Algérie.

${ }^{4}$ Laboratoire de LCGE, Faculté de Génie Mécanique, USTO-MB, Oran, Algérie.

Received: 24 July 2016 / Accepted: 12 December 2016 / Published online: 01 January 2017

\begin{abstract}
Concretes and mortars are subjected to several kinds of shrinkage strains which represent the volumic variations resulting from the cement hydration and are governed by various physical and chemical aspects. The use of polyethylene terephthalate PET plastic wastes which are available in quantity and within low cost in our country yields a very important economic and environmental impact in the construction industry. Thus, we are interested to investigate the effect of PET volumetric additive amounts for cement substituting and for the behavior of the total, drying and autogenous shrinkage. Comparison study of obtained experimental results with codale prediction models were performed according to Eurocode 2 (EC2) in order to analyze the evolution of shrinkage strain with PET-mortar composite ages and for several rates of PET waste additions. According to obtained results, PET additions acts to reduce shrinkage strains of PET-mortar composites which promote the use of these modified mortar Eco-materials in the field of construction industry.
\end{abstract}

Keywords: Composite Eco-materials; Cement substitution; Shrinkage strain; PET waste; EC2.

Author Correspondence, e-mail: amre20022000@ yahoo.fr

doi: http://dx.doi.org/10.4314/jfas.v9i1.10 


\section{INTRODUCTION}

L'utilisation de matériaux plastiques recyclés est devenue un enjeu majeur du Développement Durable, respectueux de l'Environnement. Le choix des matériaux utilisés a une répercussion directe sur la consommation en matières premières et en énergie [1,2]. Ainsi, l'utilisation de matières premières alternatives, comme les déchets plastiques exemple le polyvinyle de chlorure (PVC) [3], PET [4-10], les fibres de PET [11], le polyéthylène à haute densité (PEHD) [12], de la mousse de polyuréthane rigide [13], apporterait une réponse au problème d'épuisement des ressources naturelles.

Le polytéréphtalate d'éthylène (PET) est largement utilisé dans les bouteilles de boisson et d'autres produits d'emballages. Ainsi, l'une des méthodes logiques pour traiter les déchets plastiques de PET est de les recycler pour un usage industriel. Aujourd'hui, plusieurs projets de recherche s'intéressent sur la transformation des déchets de bouteilles de PET pour l'usage de la construction $[14,15]$.

Le retrait du béton est souvent l'origine de la fissuration, de pertes de précontrainte, d'une redistribution des contraintes, et même, plus rarement, de la ruine de l'ouvrage [16]. Cette variation dimensionnelle dépend de plusieurs paramètres tels que la composition du béton, la qualité de ses constituants, la taille des éléments ainsi que les conditions extérieures de conservation.

En général, le retrait est la diminution de volume d'un matériau ou d'un sol, due à sa dessiccation [17]. On distingue parmi les cinq grandes classes de retrait : le retrait endogène, exogène et total. Le retrait endogène est provoqué par des phénomènes internes au matériau, en l'absence de tout échange (d'eau) avec le milieu extérieur. Il est dit aussi retrait d'hydratation ou retrait d'auto-dessiccation [17] car il apparait par la densification du réseau de pores plus petits qui extraient l'eau de pores plus grands. Ce réseau de pores subit alors une dépression et provoque une diminution de volume par dessiccation interne [17].

Le retrait exogène, retrait de séchage ou retrait de dessiccation, est la conséquence directe de l'évaporation de l'eau vers l'extérieur du matériau. D'où, des tractions à l'intérieur des pores capillaires qui aboutiront au retrait [17].

Le retrait total (déformation totale) dû au retrait cs est la somme de la déformation due au 
retrait exogène cd et de la déformation due au retrait endogène ${ }_{c a}$ : d'où $c s=c d+c a[17]$.

Le but de ce travail est de présenter les résultats d'une première étude expérimentale portant sur le comportement de déformation instantanée (retrait) des matériaux composites mortier-PET qui sont élaborés à base de polytéréphtalate d'éthylène (déchets de bouteilles en PET) broyé comme substituant au ciment afin d'assurer un développement durable des Eco-matériaux de construction. Certaines proportions clés sont également étudiées, contrairement à ce qui a été entrepris dans les travaux antérieurs [5, 15, 18-22] afin de déterminer les limites de faisabilité. En outre, une approche basée sur les prédictions normatives (Eurocode 2) est incorporée dans le but de situer les résultats de déformation de retrait total obtenus expérimentalement par rapport aux modèles proposés par la reglementation européene en vigueur.

\section{MATERIAUX UTILISE}

\subsection{Le Ciment}

Le ciment utilisé dans tous les essais est un ciment CPA-CEMI 42.5 provenant de la cimenterie de la Farge, selon la Norme algérienne NA442 [23]. Les résistances minimales garanties à 02 jours $08 \mathrm{MPa}$ et à 28 jours $40.0 \mathrm{MPa}$.

Les compositions chimiques du ciment et minéralogiques du clinker sont données dans les Tableaux 1 et 2 .

Tableau 1. Composition chimique élémentaire du ciment CPA-CEM I 42.5.

\begin{tabular}{cccccccccc}
\hline Eléments & $\mathrm{SiO}_{2}$ & $\mathrm{Al}_{2} \mathrm{O}_{3}$ & $\mathrm{Fe}_{2} \mathrm{O}_{3}$ & $\mathrm{CaO}$ & $\mathrm{MgO}$ & $\mathrm{K}_{2} \mathrm{O}$ & $\mathrm{SO}_{3}$ & $\mathrm{Na}_{2} \mathrm{O}$ & $\mathrm{CaO}$ libre \\
\hline CEM I (\%) & 21.0 & 4.0 & 7.0 & 64.8 & 0.9 & 0.41 & 2.71 & 0.13 & 1.20 \\
\hline
\end{tabular}

Tableau 2. Composition minéralogique du clinker (\%).

\begin{tabular}{llll}
\hline $\mathbf{C}_{3} \mathbf{S}$ & $\mathbf{C}_{\mathbf{2}} \mathbf{S}$ & $\mathbf{C}_{\mathbf{3}} \mathbf{A}$ & $\mathbf{C}_{\mathbf{4}} \mathbf{A F}$ \\
\hline 51.30 & 25.70 & 2.80 & 14.30 \\
\hline
\end{tabular}

\subsection{Les déchets plastiques à base du PET}

Le déchet plastique du polymère utilisé est le polytéréphtalate d'éthylène connus sous le nom 
commercial PET; provenant de l'usine Tramaplaste (Tlemcen, Algérie) avec une absorption d'eau (0\%). Le PET est obtenu par le broyage très fin des bouteilles de boisson (Figure 1), ces propriétés mécaniques et thermiques sont exhibées par le Tableau 3. La courbe granulométrique est représentée dans la Figure 2. Après plusieurs essais préliminaires tels que l'analyse granulométrique des divers déchets plastiques à base de PET et l'étude des résistances mécaniques des mortiers composites avec différentes fractions granulaires (telles que la résistance à la compression et à la flexion à court, moyen et à long terme), on a décidé d'utiliser les particules du polymère inférieur à $1 \mathrm{~mm}$. La fraction granulaire supérieure à 1 mm servira comme substitution du sable par le déchet plastique au lieu du ciment pour une éventuelle seconde étude. Mais, dans notre cas, on a éliminé cette fraction à cause de la chute importante des résistances mécaniques.
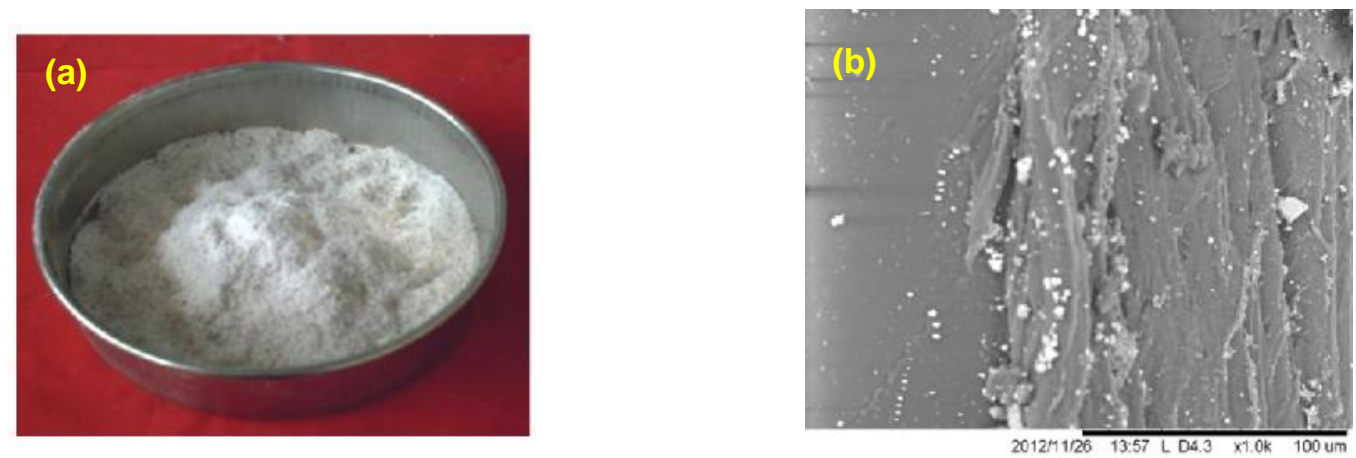

Fig. 1. (a) Photo optique, (x10) ; (b) Photo MEB des particules de PET, (x1.0k).

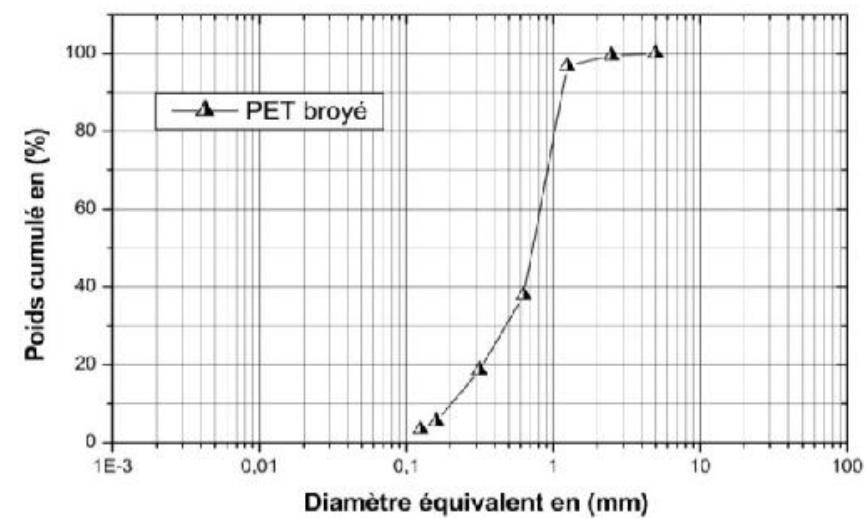

Fig. 2. La courbe granulométrique du PET broyé 
Tableau 3. Propriétés du PET utilisé

\begin{tabular}{ll}
\hline \multicolumn{2}{c}{ Propriétés Mécaniques et } \\
\hline Point de fusion & $254{ }^{\circ} \mathrm{C}$ \\
\hline La conductivité thermique & $0.13 \mathrm{~W} / \mathrm{mK}$ \\
\hline La résistance à la traction & $70 \mathrm{MPa}$ \\
\hline L'allongement à la rupture & $70 \%$ \\
\hline Le module de flexion (rigidité) & $2.0 \mathrm{MPa}$ \\
\hline Module de traction & $2.9 \mathrm{GPa}$ \\
\hline
\end{tabular}

\subsection{Le Sable utilisé}

C'est le sable de mer de Terga corrigés avec 40 \% Sable de Mer, 60 \% Sable de Carrière. Le sable est initialement préparé pour être classé suivant les normes françaises NF P15-403 [24]. Le sable est un squelette granulaire qui a le plus d'impact sur les qualités du béton et du mortier [25]. Ces propriétés physiques sont exposées par la Tableau 4.

Tableau 4. Caractéristiques physiques du sable

\begin{tabular}{|c|c|}
\hline Masse Volumique absolue $\left(\mathrm{g} / \mathrm{cm}^{3}\right)$ & 2.64 \\
\hline Masse Volumique apparente $\left(\mathrm{g} / \mathrm{cm}^{3}\right)$ & 1.44 \\
\hline Equivalent de sable (\%) & 98.4 \\
\hline Module de finesse & 1.80 \\
\hline Coefficient de courbure & 1.20 \\
\hline Coefficient d'uniformité & 2.40 \\
\hline Nature du sable & Quartzeux \\
\hline
\end{tabular}

\section{METHODES D'ESSAIS}

Afin de bien valoriser les déchets plastiques à base du PET, on procède à la quantification de leurs effets sur le comportement de déformation (retrait) des composites mortier-PET dues à la dessiccation. Les mesures des déformations concernent les éprouvettes séchant à l'air libre pour la mesure du retrait de dessiccation (Fig. 3a) et les éprouvettes isolées pour la mesure du retrait endogène (Fig. 3b).

On décide de réaliser des mortiers Eco-composites avec différents pourcentages 
volumètriques en déchets plastiques à base de PET. Le taux de substitution en volume du ciment par le PET est de l'ordre de $0 \%, 6 \%, 12 \%$ et $17 \%$.

Les éprouvettes de mortier composites sont confectionnées avec un sable corrigé avec les proportions sable-ciment-eau de 3:1:0.5 (NF EN 196.1 [26]). Une quantité de 1.0\% d'un superplastifiant (Medaplast) a été ajoutée afin d'améliorer la maniabilité selon l'ASTM C1437 [27]. Les mélanges sont coulés dans des moules prismatique $4 \times 4 \times 16 \mathrm{~cm}^{3}$ équipés de plots de retrait en laiton et recouverts de film plastiques afin d'empêcher toute évaporation au très jeune âge. A un jour d'âge, les éprouvettes sont démoulées et préparées pour les mesures de retrait selon la norme NF P 15-433 [28].

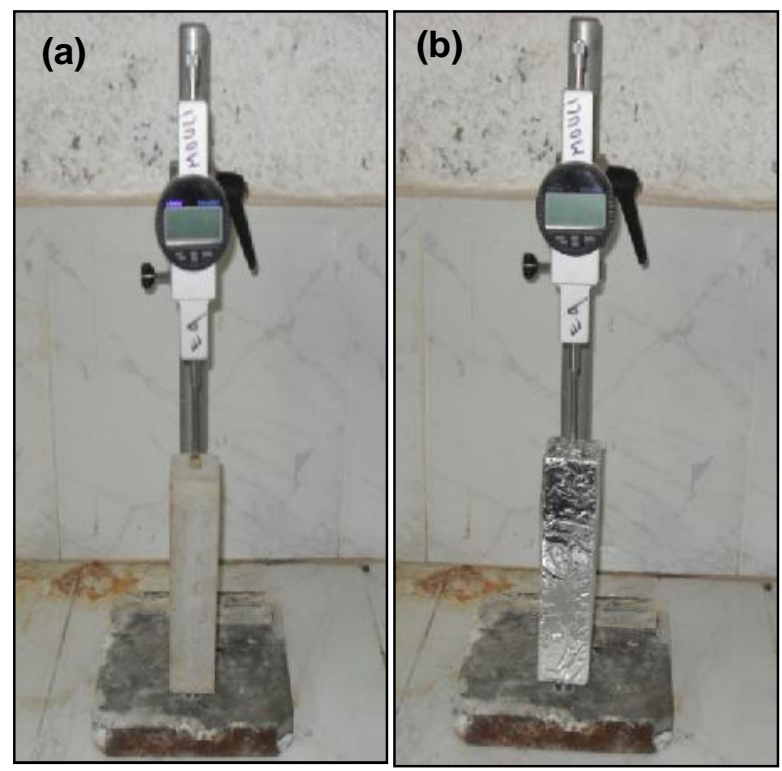

Fig. 3. Dispositif de mesure du retrait : (a) Total, (b) Endogène des Composites Mortier-PET.

L'objectif de l'essai de retrait est de mesurer, en fonction du temps, la variation de la longueur suite aux effets de l'hydratation et de la dessiccation des différents mortiers étudiés. L'expression du retrait se traduit par le rapport de la variation de longueur de l'éprouvette ( L) sur sa longueur initiale (L). Malgré la diminution de longueur des éprouvettes, ce rapport est généralement exprimé positivement en $\mathrm{m} / \mathrm{m}$ et est représenté en fonction du temps (Figure 4). 


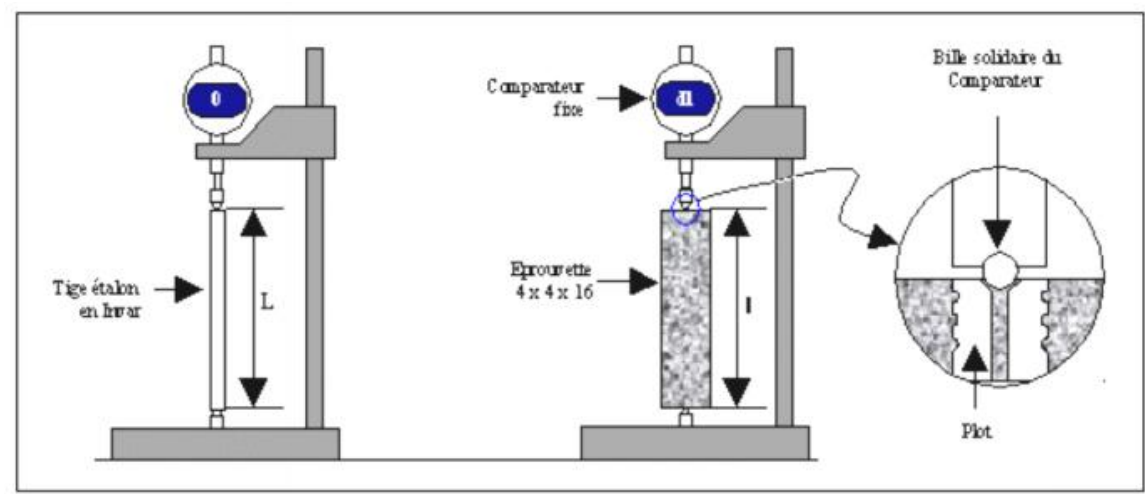

Fig. 4. Appareillage pour la mesure du retrait.

La variation relative de longueur est généralement désignée par $\varepsilon$ et a pour expression (Eq.1) :

$\varepsilon(t)=\frac{\Delta l(t)}{L}=\frac{d l(t)-d l\left(t_{0}\right)}{L}$

$\varepsilon(\mathrm{t}):$ La variation relative de longueur ;

$\Delta l(t)$ : La variation de longueur au temps $\mathrm{t} ;$

$\mathrm{L}$ : la longueur de la tige étalon soit $160 \mathrm{~mm}$;

$d l\left(t_{0}\right)$ : la longueur de l'éprouvette au temps $t_{0}$, elle est prise au moment du démoulage, soit après $24 \mathrm{~h}$ de la confection des éprouvettes ;

$d l(t)$ : la valeur lue sur le comparateur au temps $\mathrm{t}$.

En plus, les éprouvettes de retrait soumises à la dessiccation sont pesées afin de mesurer l'influence des mouvements d'eau sur les déformations. Les pertes de masses constatées pendant le durcissement correspondent à l'évaporation de l'eau libre par suite de la différence d'hygrométrie avec l'ambiance extérieure. La variation de la perte de masse est donnée par la relation suivante (Eq. 2):

Perte de masse $(\%)=\frac{\Delta M}{M} \times 100$

Les mesures représentent la moyenne de trois essais sur des éprouvettes $(4 \times 4 \times 16) \mathrm{cm}^{3}$. Après démoulage, les éprouvettes destinées à la conservation en équilibre hygrométrique sont confinées avec du papier aluminium, les autres sont exposées à l'air libre. L'ensemble doit être conservé sous température ambiante $\left(\mathrm{T}=20 \pm 2^{\circ} \mathrm{C}\right)$ et humidité relative $(\mathrm{HR}=50 \pm 5 \%)$. 


\section{RESULTATS ET DISCUSSION}

\subsection{Mesure de retrait total, endogène et exogène}

La Figure 5 illustre les résultats du retrait total pour différents taux de PET, elle montre une importante déformation du retrait total du mortier à base du PET Mais qui reste inferieur au mortier témoin cette évolution est due à l'hydratation et la dessiccation avec le milieu extérieur, néanmoins le retrait du mortier témoin se distingue des mortiers à base de PET par son évolution plus rapide à cours terme.

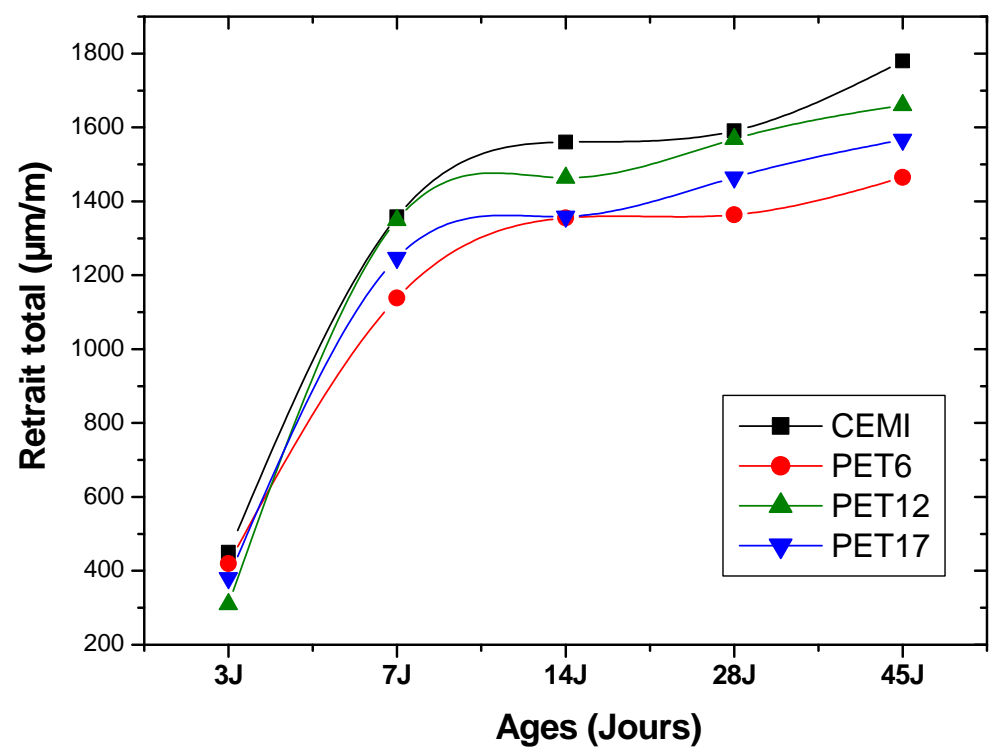

Fig. 5. Evolution du retrait Total des Composites Mortier-PET.

Le retrait endogène est la déformation des éprouvettes isolées sans échange hydrique avec le milieu extérieur. Ces valeurs témoignent de l'évolution de l'hydratation et la dessiccation interne due à la consommation d'eau par le processus chimique de la formation des hydrates. La Figure 6 illustre l'évolution du retrait endogène pour différents taux de PET avec un rapport $\mathrm{E} / \mathrm{C}=0.5$ constant. Elle présente une déformation importante à partir du septième jour mais les valeurs du retrait endogène des composites mortier-PET restent inferieures à celles du mortier témoin (CEMI) à cause de la non activité du PET qui montre un effet passif et d'autre part à l'effet hydrophobe du PET où la capacité de ce dernier contenu dans le composite à repousser l'eau (effet hydrofugeant et l'absorption d'eau du PET=0\% [29]). 


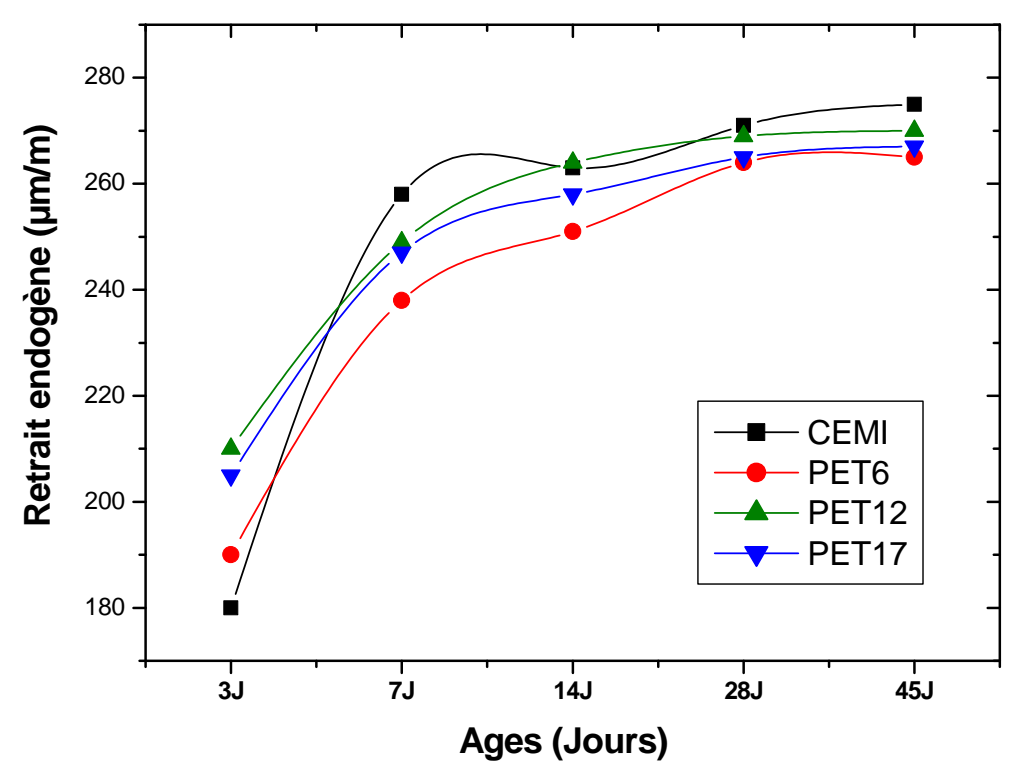

Fig. 6. Evolution du retrait Endogène des Composites Mortier-PET.

Le retrait exogène représente la déformation des éprouvettes en présence d'un échange hydrique avec le milieu extérieur. Ces valeurs représentent la combinaison entre la dessiccation et la carbonatation qui témoignent de la porosité de la microstructure et sa capacité à favoriser le séchage. Il est mesuré comme la différence des retraits de deux éprouvettes l'une libre (retrait total) et l'autre isolée (retrait endogène). La Figure 7 illustre les résultats pour l'ajout en PET et leurs taux de substitution utilisés.

Le retrait exogène représenté par la Figure 7 montre un effet positif quelque soit la quantité du PET ajouté en substitution dans le mortier. Il est caractérisé par une réduction du retrait des composites mortier-PET par rapport au mortier Témoin CEMI. Ceci est également observé d'une manière significative pour le cas des différents retraits concernant les composites PET6 et PET17 à partir de 14 jours.

Notons bien, aussi, que la substitution du ciment par les déchets plastiques à base des particules en PET exerce une influence sur le comportement de déformation des mortiers composites en diminuant, ainsi, les différents retraits des matériaux mortier-PET. Le module d'élasticité des particules en PET $\left(\mathrm{E}_{\mathrm{PET}}=2.7 \mathrm{GPa}\right)$ qui est inférieur à celui du ciment de référence CEMI [30], pourrait être utilisé pour expliquer cette diminution. Ces résultats sont en accords avec ceux trouvés par Marzouk et al. [18]. 


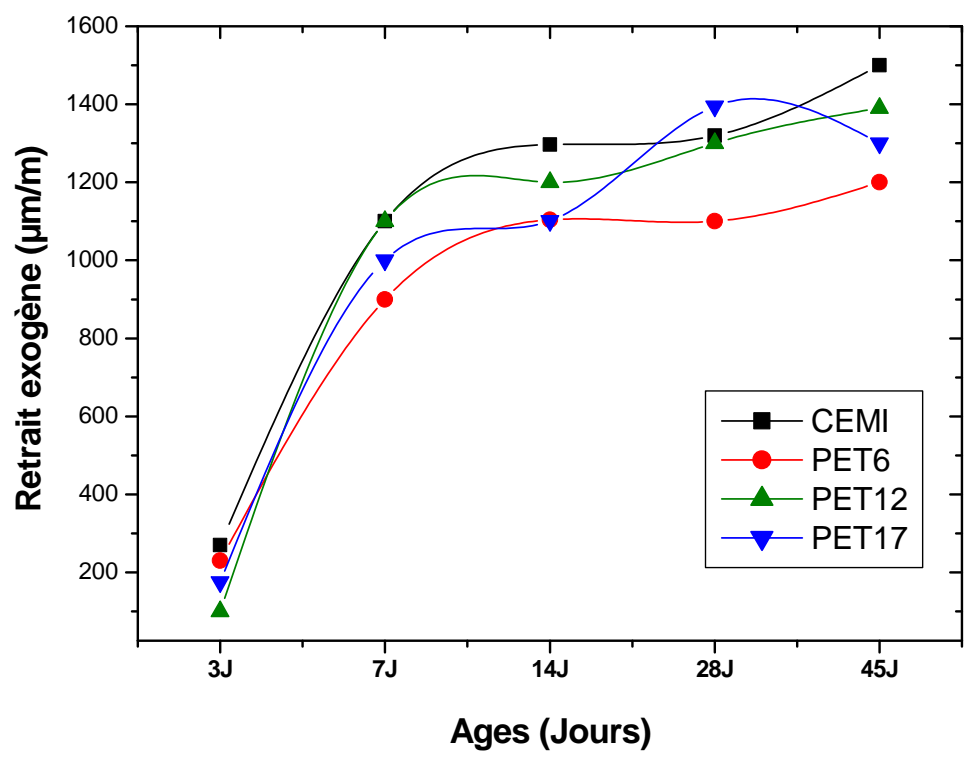

Fig. 7. Evolution du retrait Exogène des Composites Mortier-PET.

\subsection{Prédiction de déformation de retrait selon l'Eurocode2}

L'Eurocode 2 (EN 1992-1-1:2004) [31] propose un modèle indiquant l'évolution des déformations dues au retrait en fonction de la classe de ciment utilisé, la résistance moyenne à la compréssion, l'humidité relative, le rayon moyen du moule ainsi que l'age du mortier. Ce modèle a été appliqué pour étudier la tendance de la variation des déformations instantanées dues au retrait total des composites mortiers à base de PET à court terme et ceci, pour diffrentes teneurs de dechets plastiques.

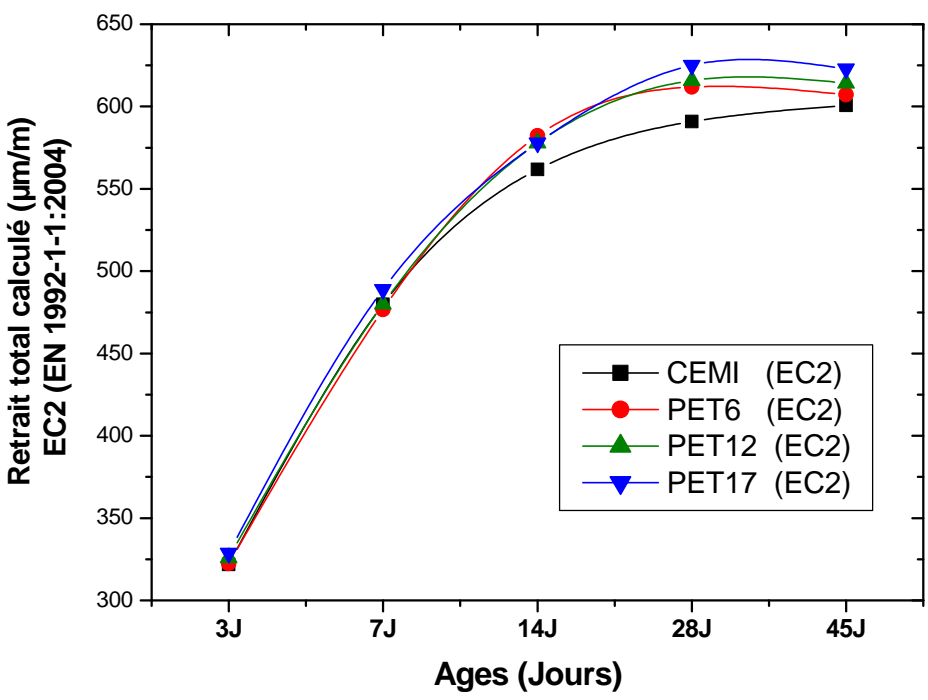

Fig. 8. Evolution de déformation dues au retrait total des Composites Mortier-PET selon les prédictions de l'EC2 (EN 1992-1-1:2004). 
La Figure 8 montre l'évolution de la déformation de retrait total selon l'EC2 (EN 1992-1-1:2004) [31] qui suit une tendence croissante en fonction de l'age du mortier pousuivie par une stabilité significative à partir de 28 jours ce qui rejoint la tendence constatée à partir des résultats expérimetaux obtenus. En termes de teneur en PET, les déformations instantanées dues au retrait augmentent avec le pourcentage d'ajout des dechets polymères contrairement ce qui a été constaté à partir des résultats expérimentaux, Figure 5. Par contre, les valeurs de déformations de retrait total calculées par l'EC2 à 03 jours, sont en bonne concordance avec celles obtenues expériementalement, Figure 9. Le tableau 5, ci-dessous, représente les grandeurs statistiques utilisées pour la prédiction des déformations dues au retrait total par le modèle EC2.

Cette observation n'est pas satisfaisante pour traduire les effets de retrait total des composites mortier-PET par ce que le modèle proposé par l'EC2 est destiné pour la prédiction des déformations de retraits des bétons, par conséquent, le modèle proposé par l'Eurocode 2 [31] n'est pas recommandé pour prédire les déformations de retrait des mortiers composites modifiés par les ajouts de PET. En plus, par ce que la formulation de ces composites Mortier-Polymère est différente de celle des bétons notamment, par rapport à l'absence d'agrégats rigides de fraction granulaire supérieure à $3 \mathrm{~mm}$ (graviers $3 / 8,8 / 15$ et 15/25) qui jouent un rôle très important pour s'opposer à l'évolution des déformations dues au retrait et qui sont pris en considération dans le modèle proposé par l'EC2 [31]. Enfin, il est important de noter que l'utilisation de l'approche proposée par l'EC2 est due à la non-existence d'un modèle normatif pour la prédiction du retrait pour ce type de mortier à base de déchets plastiques (PET). 


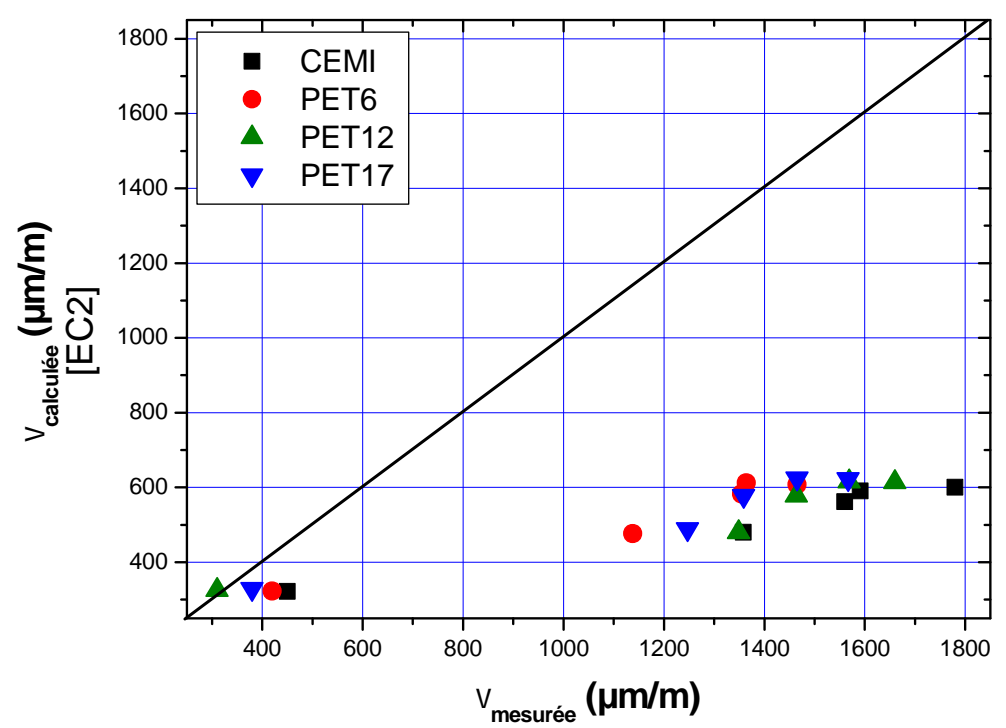

Fig. 9. Comparaison entre les valeurs expérimentales de déformation de retrait total avec celles calculées par le modèle EC2.

Tableau 5. Grandeurs statistiques pour le modèle EC2

\begin{tabular}{ccccc}
\hline EC2 & CEMI & PET6 & PET12 & PET16 \\
\hline $\begin{array}{c}\text { Courbe de } \\
\text { tendance }\end{array}$ & $\mathrm{y}=0.2174 \mathrm{x}+218.02$ & $\mathrm{y}=0.2841 \mathrm{x}+193.8$ & $\mathrm{y}=0.2153 \mathrm{x}+249.25$ & $\mathrm{y}=0.2542 \mathrm{x}+222.7$ \\
\hline $\begin{array}{c}\text { Coefficient de } \\
\text { corrélation, } \mathrm{R}^{2}\end{array}$ & 0.964 & 0.956 & 0.922 & 0.941 \\
\hline$\varepsilon_{\text {calculée }} / \varepsilon_{\text {mesurée }}$ & 0.715 & 0.768 & 1.051 & 0.865 \\
\hline \multicolumn{4}{c}{$\varepsilon:$ La variation relative de longueur (retrait). }
\end{tabular}

D'autres tentatives ont été menées pour étudier l'évolution des déformations de retrait total des mortiers composites par le biais des modèles proposés par le code ACI209R [32]. Les résultats de calculs ont montrés que ces modèles issus des essais sur les bétons ne peuvent pas être en corcordance pour le cas des mortiers composites à base de PET et ceci, en terme de précision des valeurs de déformations calculées et aussi par rapport aux teneurs d'ajout de dechets plastiques.

\subsection{Perte de masse des composites (\%)}

Les courbes de la Figure 10, montrent l'évolution moyenne des quantités d'eau évaporées en fonction de l'âge pour l'ensemble des mortiers confectionnés et conservés à l'air libre (éprouvettes non enrobées).

Nous distinguons deux étapes différentes pendant cette évolution:

- Une évaporation d'eau est accélérée jusqu'à 28 jours, 
- Au-delà de cet âge, elle commence à se stabiliser.

Ceci est dû durant la première étape, à la consommation de l'eau d'hydratation du ciment qui conduit à un assèchement interne, donc moins d'eau évaporable pendant la deuxième étape.

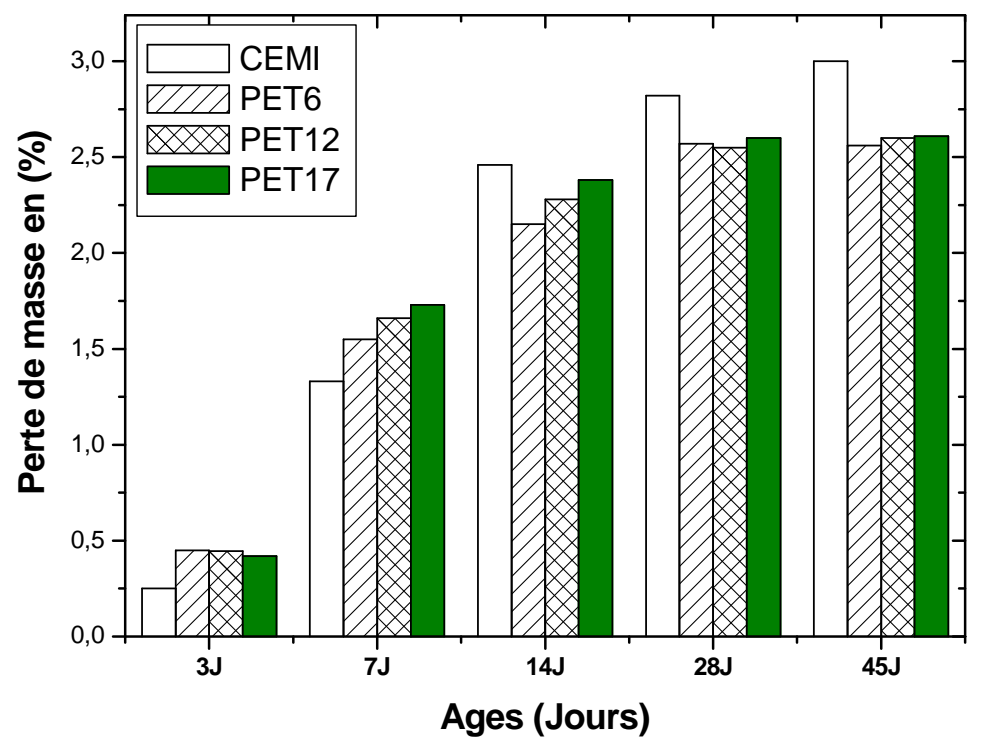

Fig. 10. Perte de masse en fonction du taux du PET (l'air libre).

Pour ce qui est des éprouvettes enrobées, Figure 11, nous distinguons une absence totale d'échange avec le milieu extérieur en termes de température et d'humidité ce qui permet uniquement à un assèchement interne du processus de réaction d'hydratation du ciment.

La Figure 11 montre l'évolution rapide de la consommation d'eau par l'effet de réaction d'hydratation du mortier de ciment à base d'ajout de PET. Contrairement au ciment de référence CEMI, plus le taux d'ajout est important plus la consommation d'eau est rapide (l'évaporation d'eau est élevée). 


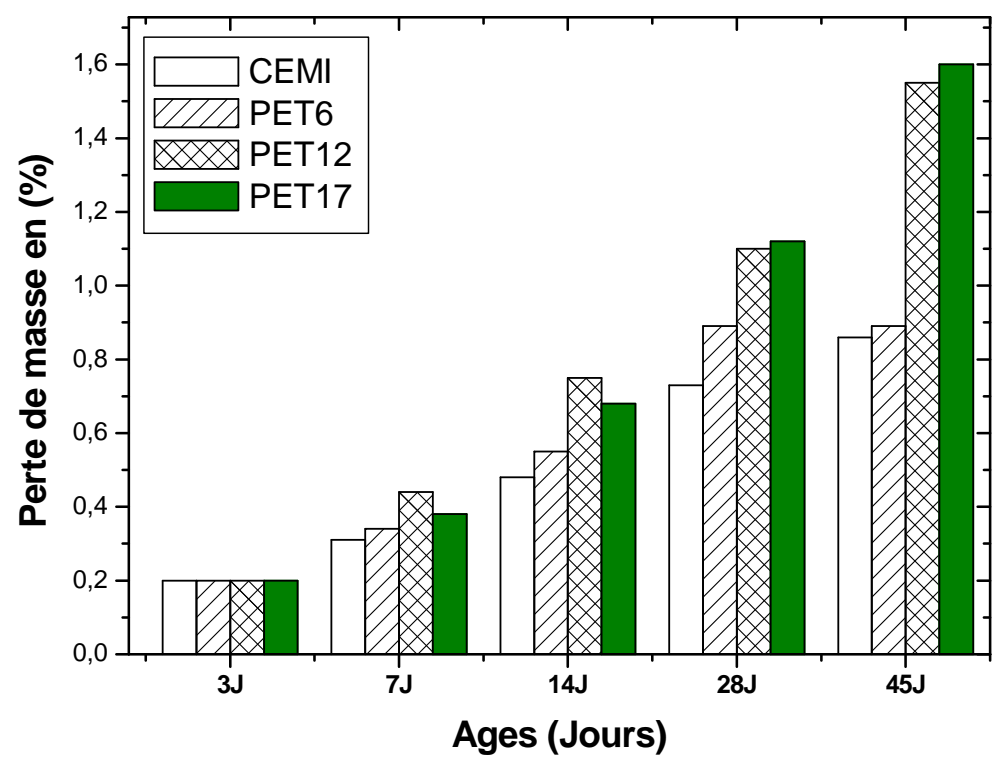

Fig. 11. Perte de masse en fonction du taux du PET (Endogène).

Puisqu'on a un remplissage des pores par les particules en PET [19], une diminution des coefficients de sorptivité des composites mortier-PET [29] et une quantité d'eau qui est adsorbée à la surface rugueuse du PET (effet écran), Figure 1b. Donc, ceci va ralentir l'hydratation du ciment par l'effet de cet ajout d'où une diminution du retrait.

\section{CONCLUSION}

La prise en compte de l'impact des déchets plastiques sur l'environnement est de plus en plus présente dans l'ensemble des secteurs d'activités, particulièrement dans celui du BTP. L'analyse des résultats de l'expérimentation nous amène à conclure que:

- L'utilisation des déchets plastiques à base du PET a un intérêt majeur pour la valorisation de ces Eco-matériaux dans le génie civil.

- Le taux d'ajout en déchets plastiques, PET, conduit à la diminution des déformations instantanées dues au retrait des mortiers composites à savoir le retrait total, endogène et exogène.

- La prédiction de ces déformations par le biais de l'Eurocode 2 n'est pas satisfaisante puisqu'elle donne des résultats ne traduisant pas les points expérimentaux particulièrement, par rapport aux effets de teneur en PET.

- L'utilisation du PET dans le mortier apporte une amélioration significative pour le 
retrait. D'où la possibilité d'utiliser ces composites Mortier-PET comme matériaux de second œuvre pour les opérations de crépissage et la fabrication des élements préfabriqués destinés aux usages généraux (trotoires, les planchers, les éléments de structure relativement minces...etc.).

L'application des éco-composites mortier-PET dans le domaine de la construction, semble réalisable compte tenu des résultats obtenus par l'analyse de ses propriétés. Potentiellement, les déchets plastiques à base de PET peuvent être un matériau de construction durable et en même temps résoudre le problème de l'environnement par le recyclage des déchets solides. En perspectives, il est intéressant de faire la substitution du sable par le déchet plastique en PET au lieu du ciment et d'entamer une deuxième étude sur le béton à haute performance qui peut développer un grand retrait d'auto-dessiccation.

\section{REMERCIEMENTS}

Ce travail a été effectué avec l'apport financier du Ministère de l'Enseignement Supérieur et de la Recherche scientifique de l'Algérie, sous les subventions du projet CNEPRU B00L01UN310120130068 (E01820120116).

\section{REFERENCES}

[1] Bourdot A., Tran Le A.D., Promis G., Douzane O., Benazzouk A., Rosquoët F. and Langlet T., Simulation Numérique du Comportement Thermique de Blocs de Construction à Base de Granulats Carbonatés. XII ${ }^{\text {̀me }}$ Colloque Interuniversitaire Franco-Québécois sur la Thermique des Systèmes, CIFQ2015 / ART-06-16, 8-10 juin 2015, Sherbrooke Québec, Canada.

[2] Bourdot A., Promis G., Douzane O., Benazzouk A., Tran Le A.D. and Langlet T., Comportement Mécanique d'éco-granulats Recyclés par Absorption de $\mathrm{CO}_{2}$ et des Bétons à Base de ces Granulats Carbonatés. XII ${ }^{\text {ème }}$ Congrès de Mécanique, 21-24 avril 2015, Casablanca, Maroc.

[3] Senhadji Y., Escadeillas G., Benosman A.S., Mouli M., Khelafi H., Ould Kaci S., J. Adhes. Sci. Technol. 2015, 29 (7), 625-640, doi.org/10.1080/01694243.2014.1000773

[4] Akcaözoğlu S., Akcaözoğlu K., Atis CD., Compos. Part B: Eng. 2013, 45, 721-726, doi.org/10.1016/j.compositesb.2012.09.012 
[5] Benosman A.S., Senhadji Y., M. Mouli, Key Eng. Mat. 2015, 650, 21-28, doi.org/10.4028/www.scientific.net/KEM.650.21

[6] Frigione M., Waste Manage. 2010, 30,1101-1106, doi.org/10.1016/j.wasman.2010.01.030

[7] Kim SB., Yi NH., Kim HY., Kim JHJ., Song YC., Cem. Concr. Compos. 2010, 32, 232-240, doi.org/10.1016/j.cemconcomp.2009.11.002

[8] Safi B., Saidi M., Aboutaleb D., Maallem M., Constr. Build. Mater. 2013, 43, 436-442, doi.org/10.1016/j.conbuildmat.2013.02.049

[9] Rahmani E., Dehestani M., Beygi M.H.A., Allahyari H., Nikbin I.M., Constr. Build. Mater. 2013, 47, 1302-1308, doi.org/10.1016/j.conbuildmat.2013.06.041

[10] Saikia N., de Brito J., Mater. Res. 2013, 16(2), 341-350, doi.org/10.1590/S1516-14392013005000017

[11] Foti D., Compos. Struct. 2013, 96, 396-404, doi.org/10.1016/j.compstruct.2012.09.019

[12] Aattache A., Mahi A., Soltani R., Mouli M., Benosman A.S., Mater. \& Design, 2013, 52, 459-469, doi.org/10.1016/j.matdes.2013.05.055

[13] Mounanga P., Gbongbon W., Poullain P., Turcry P., Cem. Concr. Compos. 2008, 30, 806-814, doi.org/10.1016/j.cemconcomp.2008.06.007

[14] Saikia N., de Brito J., Constr. Build. Mater. 2012, 34, 385-401, doi.org/10.1016/j.conbuildmat.2012.02.066

[15] Sharma R., Bansal P.P., J. Clean. Prod. 2016, 112, 473-482, doi.org/10.1016/j.jclepro.2015.08.042

[16] Acker P., Comportement mécanique du béton: Apports de l'approche physico chimique, Rapport de recherche des laboratoires des ponts et chaussées, 1988, $\mathrm{N}^{\circ} 152$ : 122.

[17] Dupain R., Lanchon R., Saint-Arroman J.-C., Granulats, Sols, Ciments et Bétons: Caractérisation des matériaux de génie civil par les essais de laboratoire, Casteilla 1995, 236 pages.

[18] Marzouk O.Y., Dheilly RM., Quéneudec M., Waste Manage. 2007, 27, 310-318. doi.org/10.1016/j.wasman.2006.03.012

[19] Benosman A.S., Taibi H., Mouli M., Senhadji Y., Belbachir M., Bahlouli I. and Houivet D., Effect of addition of PET on the mechanical performance of PET-Mortar Composite materials, J. Mater. Environ. Sci., 2015, 6 (2), 559-571.

[20] Silva P., de Brito J., Eur. J. Environ. Civ. En. 2016, 1-24. doi.org/10.1080/19648189.2015.1131200

[21] Demirboga R., Kan AUK., Constr. Build. Mater. 2012, 35, 730-734. 
doi.org/10.1016/j.conbuildmat.2012.04.105

[22] Silva R.V., de Brito J., Dhir R.K., Constr. Build. Mater. 2015, 77, 327-339. doi:10.1016/j.conbuildmat.2014.12.102

[23] NA 442, Ciment - Composition, spécifications et critères de conformité des ciments courants. $\mathrm{N}^{\circ}$ d'édition : $3 ; 2005$.

[24] NF P 15-403, Technique des essais. - Sable normal et mortier normal, homologuée le 31 juillet 1963.

[25] Baroghel-Bouny V., Characterization of cement pastes and concretes, methods, analyzes, interpretations, Central Laboratory for Roads and Bridges, 1994, pp. 468.

[26] NF EN 196-1, in: Méthodes d'essais des ciments - Partie 1: détermination des résistances mécaniques (April). Comité Européen de Normalisation (CEN), AFNOR, Paris, France, (2006).

[27] ASTM C1437-01, Standard Test Method for Flow of Hydraulic Cement Mortar, Annual book of ASTM standards, 2001.

[28] NF P 15-433, Méthodes d'essais des ciments - Détermination du retrait et du gonflement. Comité Européen de Normalisation (CEN), AFNOR, Paris, France, Février 1994.

[29] Benosman A.S., Mouli M., Taibi H., Belbachir M., Senhadji Y., Bahlouli I. and Houivet D., The Chemical, Mechanical and Thermal Properties of PET-Mortar Composites Containing Waste PET, Accepted in the Environ. Eng. Manag. J., 2013. Retrieved from: http://omicron.ch.tuiasi.ro/EEMJ/accepted.htm

[30] Benosman A.S., Taïbi H., Senhadji Y., Mouli M., Belbachir M. and Bahlouli M.I., Plastic Waste Particles in Mortar Composites: Sulfate Resistance and Thermal Coefficients, Accepted in the Prog. Rubber Plast. Re. J., 2016.

[31] NP EN 1992-1-1, Euro-code 2 Part 1.1: Design of concrete Structures, part 1-1: General rules and rules for buildings (227 p). Comité Européen de Normalisation (CEN), Brussels, Belgium, 2004.

[32] ACI-209.2R-08. Guide for modeling and calculating shrinkage and creep in hardened concrete. Michigan, USA: American Concrete Institute; 48 p., 2008.

\section{How to cite this article:}

Chaib O, Benosman A.S, Kazi Tani N, Senhadji Y, Mouli M, Taïbi H, Hamadache M. The Evolution of Shrinkage Strain of PET-Mortar Composite Eco-Materials. J. Fundam. Appl. Sci., 2017, 9(1), 136-152. 